\title{
Voltaire, Lettres philosophiques
}

\section{Franco Piva}

\section{OpenEdition}

\section{Journals}

\section{Edizione digitale}

URL: http://journals.openedition.org/studifrancesi/5962

DOI: 10.4000/studifrancesi.5962

ISSN: 2427-5856

\section{Editore}

Rosenberg \& Sellier

\section{Edizione cartacea}

Data di pubblicazione: 1 mai 2011

Paginazione: 173-174

ISSN: 0039-2944

\section{Notizia bibliografica digitale}

Franco Piva, «Voltaire, Lettres philosophiques», Studi Francesi [Online], 163 (LV | I) | 2011, online dal 30 novembre 2015, consultato il 08 janvier 2021. URL: http://journals.openedition.org/studifrancesi/5962 ; DOI: https://doi.org/ERREUR PDO dans /localdata/www-bin/Core/Core/Db/Db.class.php L.34 SQLSTATE[HY000] [2006] MySQL server has gone away

Questo documento è stato generato automaticamente il 8 janvier 2021.

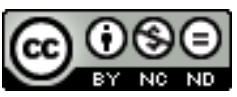

Studi Francesi è distribuita con Licenza Creative Commons Attribuzione - Non commerciale - Non opere derivate 4.0 Internazionale. 


\title{
Voltaire, Lettres philosophiques
}

\author{
Franco Piva
}

\section{NOTIZIA}

VOLTAIRE, Lettres philosophiques. Edition critique par Olivier FERRET et Anthony MCKENNA, Paris, éditions Classiques Garnier, 2010 («Bibliothèque du XVIII ${ }^{e}$ siècle», 1), pp. 604.

1 La «Bibliothèque du XVIII ${ }^{\mathrm{e}}$ siècle» voluta dai responsabili delle nuove Éditions Classiques Garnier, non poteva essere inaugurata da un'opera più prestigiosa e rappresentativa di quel secolo dei Lumi che le Lettres philosophiques di Voltaire per molti versi aprono e segnano, comunque, della loro corrosiva novità. Al punto che dopo quasi tre secoli dalla loro prima comparsa in libreria, esse non hanno ancora finito di interrogare coloro che vi si sono via via accostati; come se ogni edizione fosse destinata ad apportare elementi tali da indurre ad una nuova lettura dell'opera. Ne è una prova ulteriore l'edizione approntata da Olivier Ferret e da Anthony McKenna. A cominciare dall'«Introduction», firmata da Anthony McKenna e impostata tutta sulla presenza, alla fine delle Lettres sur l'Angleterre, della celebre Lettera XXV, qui costantemente presentata sotto l'etichetta di Anti-Pascal, e sul significato che questa presenza ha nel contesto delle Lettres e del pensiero del loro autore. Non soltanto, A. McKenna ritraccia la storia della problematica inserzione di questa Lettera all'interno delle Lettres philosophiques, ma tutto sembra girare attorno a questa Lettera: dalla storia della sua composizione, al senso che essa riveste in sé, nel percorso intellettuale di Voltaire, e nell'economia generale dell'opera. A questo fine il curatore ha ricostruito in maniera molto dettagliata il clima fortemente anti-pascaliano che caratterizzò gli anni che precedettero la composizione della Lettera, la grande attenzione che Voltaire pose alla filosofia inglese, in particolare a Locke, ma anche a quella che veniva sviluppandosi in quegli anni in tutta una serie di trattati clandestini di cui McKenna è, come è noto, un profondo conoscitore. Ne esce una visione delle Lettres e del suo autore indubbiamente nuova, e tale da spiegare certe contraddizioni che la critica non aveva mancato di sottolineare; ci pare però anche che dalla lettura proposta da McKenna esca una visione delle Lettres un po' ridotta se non riduttiva. Se l'Inghilterra apparve a Voltaire come la 
patria della tolleranza, essa rappresemtò per lui anche la patria della libertà e dei nuovi valori legati all'intelligenza delle persone e all'utilità dello Stato. Insistere troppo sull'Anti-Pascal rischia, a nostro avviso, di indirizzare su un'unica direzione la lettura di un'opera che appare invece di una grande complessità e di una straordinaria apertura.

Come è del resto provato dalle annotazioni e dai testi che accompagnano l'edizione propriamente detta, basata come è ormai tradizione sul testo pubblicato dallo stampatore-libraio Jore a Rouen nel 1734, ma data, come è uso nella collana in cui essa è presentata, in una grafia interamente ammodernata, e corredata da un significativo «choix de variantes». Il sistema annotativo che l'accompagna nasce dalla convinzione dei curatori che l'essenziale delle Lettres philosophiques più che dalle osservazioni personali di Voltaire o dalle conversazioni da lui avute con questo o quel testimone della realtà inglese, provenga da fonti scritte, «qu'il s'agisse d'ouvrages imprimés ou bien de la presse»; che l'opera, in altri termini, sia meno un reportage sull'Inghilterra che il tentativo di Voltaire di dare un fondamento serio alle proprie idee, alla luce della scoperta della filosofia inglese certo, ma più in generale del clima che andava allora diffondendosi nella parte più éclairée della «dimension philosophique du texte de même que, comme l'Introduction générale s'efforce de le montrer, sur la cohérence et la consistance que lui confère l'adjonction de l'Anti-Pascal», secondo un procedimento che con le loro stesse parole possiamo così sintetizzare: «D'une part, dans une notice liminaire [ad ogni Lettera] au cours de laquelle nous ne nous interdisons pas la pratique $\mathrm{du}$ commentaire, nous avons fourni des indications relatives au contexte intellectuel des questions abordées, éventuellement assorties d'un état des lieux qui, à propos de certaines questions centrales, prend occasionnellement la forme de développements séparés. D'autre part, les notes qui suivent se proposent d'expliciter les allusions et les références implicites, mais aussi de donner les informations essentielles sur les personnages mentionnés par Voltaire [...] Nous avons enfin opéré une série de rapprochements et de mises en perspectives»; un sostanzioso corpus di «Appendices et Annexes» offre inoltre al lettore quei testi (come la prima versione della Lettre XIII «Sur M. Locke» o il saggio intitolato $\mathrm{Du}$ suicide del 1729) che possono aiutare a ricostruire il cammino intellettuale che ha portato Voltaire a comporre le Lettres phlosophiques, oppure (è il caso degli estratti della Correspondance dati in Annexe I) che consentono di seguire il suo comportamento con amici ed editori dopo la pubblicazione delle Lettres per evitare, o attutire gli strali della censura e, contemporaneamente, assicurare all'opera non solo la più ampia diffusione ma anche la migliore interpretazione.

3 Una «Bibliographie» non esaustiva ma razionale completa questa edizione destinata ad occupare un posto di rilievo nel panorama delle edizioni critiche recenti, ma anche, ne siamo certi, a far discutere per il taglio con il quale è stata condotta e per la lettura che dell'opera di Voltaire essa propone. 